Proyecciones

Vol. 24, No 2, pp. 167-173, August 2005.

Universidad Católica del Norte

Antofagasta - Chile

\title{
STRATEGY FOR TO STABILIZE NON LINEAR SYSTEMS THROUGH DIRECTIONAL CONTROLS *
}

\author{
VÍCTOR DELGADO \\ Universidad Austral de Chile. \\ Received: June 2004. Accepted : August 2005
}

\begin{abstract}
For a non linear system, with an isolated and non asymptotically stable equilibrium point, we had obtained a control strategy which disturb the system so that the dynamic move locally towards the equilibrium point. We consider the linearization of the system and feedback directional controls.
\end{abstract}

KEYWORKS : Linearly equivalent systems, directional feedback controls.

*Supported by Dirección de Investigación U. Austral de Chile, Proyecto 200064. 


\section{Introduction}

Let $M$ be a linear manifold of $k$-codimension, $x_{0}=\left(x_{1_{0}}, \ldots, x_{n_{0}}\right) \in M$ and $x(t, u)=\left(x_{1}(t, u), \ldots, x_{n}(t, u)\right) \quad$ with $\quad x(0, u)=x_{0} \quad$ a solution of the canonical linear control system:

$$
x_{1}^{\prime}=x_{2} x_{2} \prime=x_{3} \vdots x_{n}^{\prime}=a_{1} x_{1}+\ldots+a_{n} x_{n}+u
$$

Our objective is to find necessary conditions for the control $u=u(t)$ such that the respective solution $x(t, u)$ of the system (1.1) belongs to $M$. This problem has been investigated mainly for linear control system $x \prime=A(t) x+B(t) u$ and their non linear perturbation $x \prime=A(t) x+B(t) u+$ $f(t, x, u)$ in the $n$-dimensional Euclidean space $E^{n}$ ([1], [3]). Their results are independent of the codimension of the linear manifold considered. In this sense ours results may be summarized saying that if $x(t, u)$ is a solution of system (1.1) such that belong to $M$ for non negative $t$, then $u(t)$ is a feedback control which satisfies conditions dependent of $k$. We call such $u=u(t)$ a feedback directional control

Next we consider a nonlinear system in the plane with an isolated equilibrium point which is not asymptotically stable. Our aim is to find a control strategy such that disturb the system and move the dynamic towards the equilibrium state. The natural way to realize this objective is to consider the linearization of the non linear control system. If that process give a controllable linear system then itś linearly equivalent to system (1.1) ([5], [6]system we consider the feedback directional control whose respective trajectory transfers a given initial state to the equilibrium point. Under certain conditions this feedback directional controls disturb the non linear system so that the respective dynamic move asymptotically towards the equilibrium state.

\section{Results}

Lemma 1. We denote by $\langle x, y\rangle$ the usual inner product in an Euclidean space $E^{n}$ and $A^{T}$ the transpose of a matrix. Let $x(t, u)$ be a solution of system (1.1) such that $x(t, u) \in M$ for non negative $t$, where $M$ is a linear manifold of 1-codimension. If $m_{i}=-1,1 \leq i \leq n$, is the last non zero component of the normal vector $m$ to the manifold $M$, then

$$
u=<\left(N^{n-i}\right)^{T} m, A x>
$$


where $A$ is the companion matrix of system (1.1) and $N$ is a nilpotent matrix of index $n$.

Proof. If $x(t, u)$ is a solution of system (1.1) such that $x(t, u) \in M$ then

$$
<x(t, u)-x_{0}, m>=0
$$

For $i=1$, that is $m=(-1,0, \ldots, 0)$, we obtain from $(2.2)$ the solution $x(t, u)=\left(x_{1_{0}, 0, \ldots, 0}\right)$, so $0=x_{n^{\prime}}(t, u)=a_{1} x_{1_{0}}+u$.

Therefore $u=-a_{1} x_{1_{0}}=<\left(N^{n-1}\right)^{T} m, A x>$ where $A$ is the companion matrix of system (1.1) and $N$ is a nilpotent matrix of index $n$.

For $1<i \leq n$ we obtain from (2.2): $\quad\left(x_{1}(t, u)-x_{1_{0}}\right) m_{1}+\ldots+$ $\left(x_{i}(t, u)-x_{i_{0}}\right)(-1)=0$

Using this along with (1.1) leads to:

$$
x_{n-i+2}(t, u) m_{1}+\ldots+x_{n}(t, u) m_{i-1}+x_{n}(t, u)(-1)=0
$$

Since $x_{n^{\prime}}(t, u)=<a, x(t, u)>+u(t)$ where $a=\left(a_{1}, \ldots, a_{n}\right)$, we obtain from $(2.3)$

$$
u=<m,\left(x_{n-i+2}(t, u), \ldots, x_{n}(t, u),<a, x(t, u)>, 0, \ldots, 0\right)>
$$

Now if $N$ is a nilpotent matrix of index $\mathrm{n}$ then

$$
N^{n-i} A x(t, u)=\left(x_{n-i+2}(t, u), \ldots, x_{n}(t, u),<a, x(t, u)>, 0, \ldots, 0\right)
$$

where $A$ is the companion matrix of system (1.1), i.e.:

$$
u=<m, N^{n-i} A x>=<\left(N^{n-i}\right)^{T} m, A x>
$$

Theorem 2. Let $S$ be a linear subspace of $k$-codimension, $1 \leq k<n$, and let $S^{0}$ be the orthogonal subspace generated by the vectors $m_{i}, i=1, \ldots, k$, such that its last non zero component are respectively $m_{i, n_{i}}=-1$. If $x(t, u)$ is a solution of system (1.1) and belong to $M=x_{0}+S$ for non negative $t$, then the control $u=u(t)$ satisfies

$$
u=<\left(N^{n-n_{i}}\right)^{T} m_{i}, A x>
$$

for each $i=1, \ldots, k$, where $A$ is the companion matrix of system (1.1) and $N$ is a nilpotent matrix of index $n$. 
Proof. Since $\left.<x(t, u)-x_{0}, m_{i}\right\rangle=0$ for non negative $\mathrm{t}$ and each $i=1, \ldots, k$, we can apply the above lemma for each $m_{i}$ and obtain the formula (2.4).

Example 3. The aplication of the above result in the plane gives: $M$ is a linear manifold of 1-codimension; $\left(x_{0}, y_{0}\right) \in M$ for non negative $t$ and:

$$
\begin{aligned}
& x^{\prime}=y \\
& y^{\prime}=a x+b y+u
\end{aligned}
$$

If the normal vector to $M$ is $(-1,0)$ or $(0,-1)$ then from (2) $u=-a x-b y$ and the respective solution of system (6.6) is $(x(t), y(t))=$ $\left(x_{0}, y_{0}\right)+t\left(\left(y_{0}, 0\right)\right.$; i.e. for $y_{0} \neq 0$ we obtain horizontal trajectories and for $y_{0}=0$ only stationary points.

If the normal vector to $M$ is $(m,-1)$ then from (2) $u=m y-a x-b y$ and the respective solution of system $(2.5)$ is $(x(t), y(t))=\left(x_{0}, y_{0}\right)+\left(e^{m t}-\right.$ 1) $\left(y_{0} / m, y_{0}\right)$; i.e. for $y_{0} \neq 0$ we obtain oblique trajectories and for $y_{0}=0$ only stationary points.

In conclusion for each $\left(x^{*}, y^{*}\right)$ exist a region whose points can be transfer towards $\left(x^{*}, y^{*}\right)$ through directional trajectories of system (2.5).

Theorem 4. Let $\left(x^{*}, y^{*}, 0\right)$ be an isolated equilibrium state which is not asymptotically stable for the nonlinear control system:

$$
\begin{array}{lc}
x^{\prime} & =\quad f(x, y) \\
y^{\prime} & =g(x, y)+u
\end{array}
$$

where $f, g$ are $C^{1}$ and $J=J\left(x^{*}, y^{*}\right)$ is the Jacobian with $\partial f / \partial y\left(x^{*}, y^{*}\right) \neq$ 0 . If moreover

$$
\begin{array}{rll}
\operatorname{det} J\left(1-\frac{<\nabla f\left(x^{*}, y^{*}\right),\left(x^{*}, y^{*}\right)>}{y^{*}}\right) & >0 & (\nabla=\text { gradient }) \\
<(-\operatorname{det} J, \operatorname{tr} J),\left(x^{*}, y *\right)> & <0 & (\text { tr }=\text { traza })
\end{array}
$$

then there exist a feedback directional control which disturb the system (2.6) in such a way that the equilibrium state become locally asymptotically stable. 
Proof. Since the equilibrium state is not asymptotically stable, the Jacobian matrix: $\mathrm{J}=\left[\begin{array}{ll}\partial f / \partial x & \partial f / \partial y \\ \partial g / \partial x & \partial g / \partial y\end{array}\right]_{\left(x^{*}, y^{*}\right)}$ is nonstable; therefore $\quad \operatorname{det} J \leq$ 0 or $\operatorname{tr} J \geq 0 \quad([2])$.

Now since $\partial f / \partial y\left(x^{*}, y^{*}\right) \neq 0$ the linearization of system (2.6) gives: $\left[\begin{array}{l}x \\ y\end{array}\right]^{\prime}=J\left[\begin{array}{l}x \\ y\end{array}\right]+\left[\begin{array}{l}0 \\ 1\end{array}\right] u$

which is a controllable linear system and therefore linearly equivalent to

$$
\left[\begin{array}{l}
x \\
y
\end{array}\right]^{\prime}=\left[\begin{array}{cc}
0 & 1 \\
-\operatorname{det} J & \operatorname{tr} J
\end{array}\right]\left[\begin{array}{l}
x \\
y
\end{array}\right]+\left[\begin{array}{l}
0 \\
1
\end{array}\right] u([4],[5],[6])
$$

By example 3, we must consider the family of feedback directional controls:

$$
u(x, y)=m y+(\operatorname{det} J) x-(\operatorname{tr} J) y
$$

and to choose $m$ in such a way that $u\left(x^{*} \cdot y^{*}\right)=0$.

Hence:

$$
m=\operatorname{tr} J-\left((\operatorname{det} J) x^{*} / y^{*}\right)
$$

Replace (2.8) in (2.7) and obtain a directional control that disturb the system (2.6) in the following sense:

$$
\begin{aligned}
& x \prime=f(x, y) \\
& y^{\prime}=g(x, y)+(\operatorname{det} J) x-\left((\operatorname{det} J) x^{*} / y^{*}\right) y
\end{aligned}
$$

whose Jacobian matrix $J^{*}\left(x^{*}, y^{*}\right)$ is:

$$
\left[\begin{array}{cc}
\frac{\partial f}{\partial x}\left(x^{*}, y^{*}\right) & \frac{\partial f}{\partial y}\left(x^{*}, y^{*}\right) \\
\frac{\partial g}{\partial x}\left(x^{*}, y^{*}\right) & \frac{\partial g}{\partial y}\left(x^{*}, y^{*}\right)-\left((\operatorname{det} J) x^{*} / y^{*}\right)
\end{array}\right]
$$

In consequence

$$
\begin{aligned}
\operatorname{det} J^{*} & =\operatorname{det} J\left(1-\frac{x^{*}}{y^{*}} \frac{\partial f}{\partial x}\left(x^{*}, y^{*}\right)-\frac{\partial f}{\partial y}\left(x^{*}, y^{*}\right)\right) \\
\operatorname{tr} J & =\frac{\partial f}{\partial x}\left(x^{*}, y^{*}\right)+\frac{\partial g}{\partial y}\left(x^{*}, y^{*}\right)-\frac{x^{*} \operatorname{det} J}{y^{*}}
\end{aligned}
$$

and, by hypothesis of the theorem, we obtain: $\operatorname{det} J^{*}\left(x^{*}, y^{*}\right)>0$ and $\operatorname{tr} J^{*}\left(x^{*}, y^{*}\right)<0$ Therefore the equilibrium state is locally asymptotically stable. 
Example 5. The periodic solutions of the Lotka-Volterra populations models may be disturb using feedback directional controls over the predators in such a way that the respective trajectory move asymtotically to the equilibrium point. That is:

$$
\begin{aligned}
& x \prime=a x-b x y \\
& y^{\prime}=-c y+d x y+u
\end{aligned}
$$

$a, b, c, d$ positive parameters.

$$
\begin{aligned}
& \left(x^{*}, y^{*}\right)=(c / d, a / b) \text { equilibrium state non asymptotically stable } \\
& f(x, y)=a x-b x y ; \partial f / \partial y\left(x^{*}, y^{*}\right) \neq 0 ; \nabla f\left(x^{*}, y^{*}\right)=(0,-b c / d) \\
& \operatorname{det} J\left(1-<(0,-b c / d),\left(x^{*}, y^{*}\right)>/ y^{*}\right)=a c(1+(b c / d))>0 \\
& <(-\operatorname{det} J, \operatorname{tr} J),\left(x^{*}, y^{*}\right)>=-a c^{2} / d<0
\end{aligned}
$$

Therefore by theorem 4 the feedback directional control :

$$
u=u(x, y)=(\operatorname{det} J) x-\left((\operatorname{det} J) x^{*} / y^{*}\right) y=a c x-\left(b c^{2} / d\right) y
$$

become the above system locally asymptotically stable around the equilibrium point $\left(x^{*}, y^{*}\right)$.

\section{References}

[1] Balachandran-Dauer, Controllability of Nonlinear Systems to Affine Manifolds, J. Optim.Th. and Appl., Vol 64 \#1, pp. 15-27, (1990)

[2] Beltrami E., Mathematics for Dynamic Modelling, Academic Press, Inc. (1987).

[3] Chukwu, E. N., Total Controllability to Affine Manifolds of Control Systems, J. Optim. Th. and Appl., Vol \# 42, pp. 181-199, (1864).

[4] Delgado V., Equivalencia lineal de Sistemas de Control y su interpretación en algunos modelos biomatemáticos, Memoria VII Congr. Internacional de Biomat. pp. $72-79$ Buenos Aires, (1995).

[5] Delgado V., Estabilización del modelo poblacional de May a través de controles direccionales, Rev. Mat. Th. y Apl., Cimpa, UCR, Vol 8 \# 2, pp. 79-83, (2001).

[6] Lee E. B., Markus L., Foundations of Optimal Control Theory, Longman Scientific, Technical, (1988). 


\section{Víctor Delgado}

Instituto de Matemática

Universidad Austral de Chile

Casilla 567

Valdivia

Chile

e-mail: vdelgado@uach.cl 